\title{
Análisis de los incidentes de trauma dentoalveolar atendidos en el servicio de urgencias de una institución docente asistencial de Medellín (Colombia) 2007-2012
}

\author{
Eliana María Pérez Amaya, Est. ${ }_{1}$, Lilibeth Ruiz Correa*, Est. ., Leidy Daiana Peláez Jiménez, Est., Natalia \\ Salazar Garzón, Est. ${ }_{1}$, Steven Orozco Arcila, Odont. ${ }_{2}$ Cecilia María Martínez Delgado, MsC. ${ }_{1}$
}

Recibido: 10 de febrero del 2014. Aprobado: 25 de abril del 2014.

*Autor de correspondencia: Lilibeth Ruiz, Facultad de Odontología, Universidad de Antioquia, Medellín, Colombia. Calle 64 n. ${ }^{0}$ 52-59, teléfono 2196772, correo electrónico: libeli_12@hotmail.com

Cómo citar este artículo: Pérez EM, Ruiz L, Peláez LD, Salazar N, Orozco S, Martínez CM. Análisis de los incidentes de trauma dentoalveolar atendidos en el servicio de urgencias de una institución docente asistencial de Medellín (Colombia) 2007-2012. Rev Nac Odontol. 2014;10(18):21-8. doi: http://dx.doi.org/10.16925/ od.v10i18.718

Resumen. Introducción: el trauma dental es considerado una urgencia odontológica y su frecuencia es alta principalmente en población joven. El objetivo de esta investigación fue analizar los incidentes de trauma dentoalveolar que consultan al servicio de urgencias de una clínica universitaria de Medellín (Colombia), entre los años 2007 y 2012. Métodos: estudio descriptivo retrospectivo. Se revisaron 7.555 historias clínicas, de las cuales 549 constituyeron la muestra definitiva porque cumplieron con los criterios de inclusión y exclusión. Resultados: de todas las causas de consulta en el servicio de urgencias, el trauma dentoalveolar tuvo una frecuencia de 7,3\%; el sexo femenino fue el que más consultó (53,2\%). La población entre 11 y 20 años fue la más afectada (18,4\%). Los accidentes por caídas y por actividades deportivas fueron el 15,1\% y el 6,7\% de las causas, respectivamente. Conclusión: el trauma dentoalveolar es un evento común en la consulta odontológica de urgencias.

Palabras clave: traumatismos de los dientes, fracturas dentales, accidentes, servicios de salud bucal.

Analysis of dentoalveolar trauma incidents treated in the emergency services department at a healthcare teaching institution in Medellin (Colombia) 2007-2012

Abstract. Introduction: dental trauma is considered a dental emergency and it occurs frequently, especially among young people. The aim of this research was to analyze dentoalveolar trauma incidents treated in the emergency services department of a university clinic in the city of Medellin (Colombia), between 2007 and 2012. Methods: this was a retrospective, descriptive study in which 7.555 clinical histories were revised, the final sample comprising the 549 cases that met inclusion and exclusion criteria. Results: of all causes for consultations, 7,3\% were due to dentoalveolar trauma; most patients seeking treatment were females $(53,2 \%)$. The population aged between 11 and 20 was the most affected $(18,4 \%)$. Falls and sporting activities caused $15,1 \%$ and $6,7 \%$ of accidents respectively. Conclusion: dentoalveolar trauma is a common occurrence in emergency dental treatment.

Keywords: tooth trauma, dental fractures, accidents, oral health services.
Análise dos incidentes de traumas dentoalveolar atendidos no serviço de pronto-socorro de uma instituição docente assitencial de Medellín (Colômbia) 2007-2012

Resumo. Introdução: o trauma dental é considerado uma emergência odontológica e sua frequência é alta principalmente na população jovem. O objetivo desta pesquisa foi analisar os incidentes de trauma dentoalveolar que consultam o serviço de pronto-socorro de uma clínica universitária da cidade de Medellín (Colômbia), entre os anos de 2007 e 2012. Métodos: estudo descritivo retrospectivo. Revisaram-se 7.555 prontuários, dos quais 549 constituíram a amostragem definitiva porque cumpriram com os critérios de inclusão e exclusão. Resultados: de todas as causas de consulta no serviço de pronto-socorro, o trauma dentoalveolar teve uma frequência de 7,3\%; o sexo feminino foi o que mais se consultou $(53,2 \%)$. A população entre 11 e 20 anos foi a mais afetada (18,4\%). Os acidentes por quedas e por atividades esportivas somaram $15,1 \% \mathrm{e}$ $6,7 \%$ das causas, respectivamente. Conclusão: o trauma dentoalveolar é um evento comum na consulta odontológica de emergência.

Palavras-chave: Traumatismo dos dentes, fraturas dentais, acidentes, serviços de saúde bucal. 


\section{Introducción}

El trauma dental se define como la lesión de los tejidos dentales y periodontales producida por impactos con objetos, con la mano, en caídas, golpes, actos de violencia, actividades deportivas, accidentes de tránsito, como las principales causas [1-8]. Es considerado una urgencia odontológica porque genera dolor, alteración funcional por disminución de la capacidad de masticación y dicción y molestia en el campo estético, que termina por incidir en el estado de ánimo del paciente y su calidad de vida [1-3].

Los traumatismos sufridos sobre la dentición temporal pueden lesionar los gérmenes permanentes en desarrollo, induciendo diversos grados de alteración en la formación de los tejidos duros, en el desarrollo radicular o detención de la erupción; sin embargo, un factor favorable es que los pacientes menores de 20 años poseen mayor capacidad de cicatrización que los adultos [9].

Otro aspecto relevante es la participación más activa de los niños en los juegos y actividades deportivas; los niños son más propensos que las niñas a sufrir un trauma dentoalveolar. Cuando este ocurre, se ven afectados principalmente los incisivos centrales superiores, como lo muestra el estudio del Hospital Universitario Cajuru (Brasil), con un porcentaje del 57,9\% [2]. Además, se encuentran condiciones predisponentes para el traumatismo en los niños como la amelogénesis y la dentinogénesis imperfectas [6,10-12].

En general, las cifras de ocurrencia de trauma varían de un país a otro e igualmente existen variaciones según el sexo. En Colombia, el III Estudio Nacional de Salud Bucal (1998) reportó una prevalencia del 16,7\% en edades de 7 a 15 años [3]. Otro estudio realizado en la Clínica CES (Sabaneta, Antioquia) reportó una prevalencia en el grupo etario de 0 a 5 años de 41,6\% [13], mientras que en Recife (Brasil), en edades de 1 a 7 años, la prevalencia fue de 51,9\%, con un pico en la edad de 3 años [14].

Estudios en Cajuru (Brasil) concluyeron que los hombres presentaron el 65,5\% de los traumatismos [2]. El estudio de Recife (Brasil), mencionado arriba, evidenció un porcentaje más alto en hombres (89,2\%) [14], y finalmente un estudio de la Clínica de Atención Odontológica Integral, en la Facultad de Odontología de Araçatuba (UNESP), entre enero de 1992 y julio de 2002, refiere una frecuencia del 69\% en hombres [15].

Los actos violentos, los accidentes automovilísticos, las caídas y golpes representan la mayoría de los traumas dentoalveolares atendidos en los servicios de salud; a menudo se presentan concomitantes con po- litraumatismos en diferentes áreas del cuerpo, lo que hace que la intervención odontológica del trauma dentoalveolar no sea la prioridad $[2,8]$.

En Piracicaba (Brasil), se reportó que las causas más frecuentes de trauma dentoalveolar en la población general fueron las caídas y golpes, seguidos de los accidentes ciclísticos (27,61\%), accidentes de tráfico (10,47\%) y en motocicleta $(6,40 \%)$ [16]. En pacientes adultos, la principal causa de trauma dentoalveolar fueron las agresiones, los accidentes de tránsito y las actividades deportivas [17]. Sin embargo, otro estudio realizado en Recife (Brasil), en pacientes entre 19 y 80 años, reporta que la principal causa fueron los accidentes en motocicleta [14].

Otro de los factores etiológicos del trauma dentoalveolar es el uso indebido de los dientes, al emplearlos como herramientas de trabajo, morder objetos duros o sostener dichos objetos con los dientes; sin embargo, lesiones por esta causa solo tienen una incidencia de menos del 8\% [5].

Los traumatismos más frecuentes en los dientes, según estudios reportados, fueron: fractura coronal no complicada $(54,2 \%)$ y luxación lateral $(22,7 \%)$ en el estudio realizado en la Clínica CEs de Medellín [9]; subluxación (21,46\%), avulsión (17,18\%), luxación lateral (16,66\%) en Brasil [2], y fractura no complicada de corona $(26,2 \%)$ y luxación lateral $(23,3 \%)$ en un estudio en Republica Checa [18].

En el trauma dentoalveolar, hay diferentes factores predisponentes como las maloclusiones, la cobertura labial inadecuada, la condición socioeconómica desfavorable, los aparatos de ortodoncia, las convulsiones y la anestesia general por la presencia del tubo endotraqueal $[4,5]$.

Se han propuesto diferentes maneras de clasificar los traumatismos dentales y sus estructuras de sostén, aptas para cualquier dentición y ampliamente usadas para su correcta aplicación en la práctica clínica. Para el desarrollo de esta investigación, se tuvo en cuenta la propuesta de Andreassen $[19,20]$, autor que las divide en lesiones de los tejidos duros dentarios y de la pulpa, lesiones de los tejidos periodontales y lesiones del hueso de sostén.

Ellis utiliza una descripción ampliamente usada que se basa en la extensión de la lesión, y las clasifica desde la Clase I hasta la Clase Ix [19]. Otra clasificación propuesta por Basrani es la descripción dada por las características anatómicas de las estructuras dentarias, como fracturas coronarias, fracturas radiculares y fracturas corono-radiculares.

La posibilidad de disminuir los efectos negativos del trauma dental anteriormente descritos y garantizar 
la exitosa recuperación del paciente se encuentra asociada con la atención oportuna en el menor tiempo posible, después del trauma y la calidad de la atención recibida.

Por todo lo anterior, se consideró importante reconocer la frecuencia del trauma dentoalveolar, las causas y el tipo de traumatismos más frecuentes, la distribución por sexo y edad y demás variables que pudiesen intervenir en estos eventos, en las personas atendidas en el servicio de urgencias de una clínica universitaria en el periodo comprendido entre 2007 y 2012. La información obtenida servirá para orientar de mejor manera la atención de estos pacientes.

\section{Métodos}

Se realizó un estudio descriptivo retrospectivo, basado en historias clínicas del servicio de urgencias de una clínica universitaria de Medellín, en un periodo comprendido entre los años 2007 y 2012. La recolección de la información utilizó un formato elaborado con las variables de interés para la investigación, previa prueba piloto.

El muestreo fue no probabilístico; se revisaron un total de 7.555 historias clínicas en el periodo de estudio; 549 historias se valoraron porque cumplían con los criterios de inclusión. Se excluyeron los registros que informaban fracturas por lesiones cariosas, desalojo de obturaciones y traumas anteriores.

La información recolectada se procesó en el programa Excel $2010^{\circ}$ y en el programa spss (vs.19) ${ }^{\circ}$. Los datos se analizaron de manera univariada utilizando medidas de tendencia central y dispersión (variables cuantitativas) y de frecuencias (variables cualitativas); se exploraron asociaciones estadísticas de tipo bivariado y multivariado que permitan, a futuro, investigaciones de tipo causal.

\section{Resultados}

\section{Resultados descriptivos}

La muestra total analizada fueron 549 registros de pacientes que consultaron al servicio de urgencias de una institución docente-asistencial de Medellín (Colombia), entre los años 2007 y 2012, de un total de 7.555 registros. De todas las causas de consulta en urgencias, el trauma dentoalveolar ocupó el 7,3\%.

La mayor frecuencia de consulta reportada ocurrió en el $2007(28,1 \% ; \mathrm{n}=154)$, y se observó una tendencia al aumento a partir de 2010 hasta el año 2012 (figura 1).

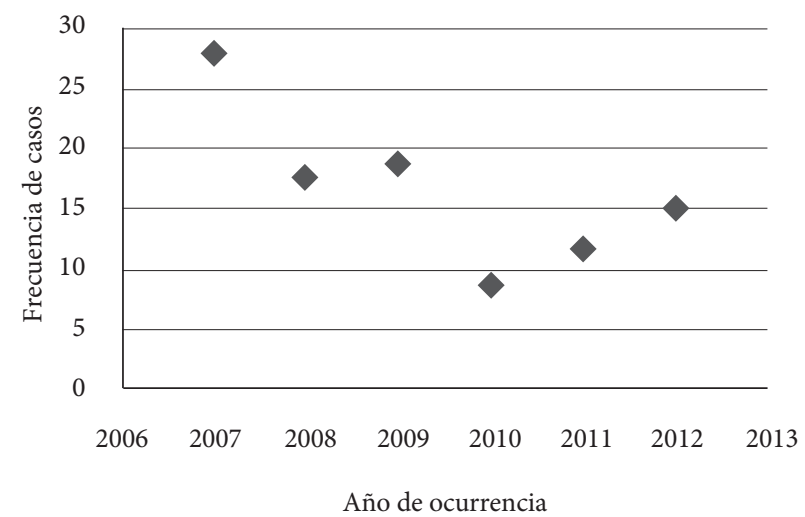

Figura 1. Frecuencia de casos de trauma dentoalveolar por año de ocurrencia. Universidad de Antioquia, Medellín, 2007-2012 Fuente: historias clínicas institucionales

Las mujeres fueron quienes más consultaron ( $\mathrm{n}=292 ; 53,2 \%$ ); por edad, todos los grupos etarios consultaron al servicio; las edades entre 11 y 20 años ( $\mathrm{n}=101 ; 18,4 \%)$ pueden tener causas diferentes de quienes tienen entre 40 y 50 años $(\mathrm{n}=103 ; 18,8 \%)$ y quienes son mayores de 50 años ( $\mathrm{n}=101 ; 18,4 \%)$, aunque la frecuencia es muy semejante entre los grupos de edad analizados.

En cuanto a la ocupación de las personas que consultaron al servicio, se observó que la mayoría de los traumatismos fueron en estudiantes ( $\mathrm{n}=151 ; 27,5 \%$; tabla 1); sin embargo, las amas de casa $(\mathrm{n}=113 ; 20,6 \%)$, los trabajadores dependientes $(\mathrm{n}=104 ; 18,9 \%)$ y los independientes $(n=64 ; 11,7 \%)$ tuvieron un porcentaje significativo.

Tabla 1. Principales ocupaciones de los consultantes al servicio de urgencias de una institución docente-asistencial. Universidad de Antioquia, Medellín, 2007-2012

\begin{tabular}{|l|c|c|}
\hline \multicolumn{1}{|c|}{ Ocupación } & $\mathbf{N .}^{\mathbf{o}}$ & $\%$ \\
\hline Estudiante & 151 & 27,5 \\
\hline Ama de casa & 113 & 20,6 \\
\hline Sin dato & 68 & 12,4 \\
\hline Desempleado & 22 & 4 \\
\hline Sin oficio & 20 & 3,6 \\
\hline Trabajador independiente & 64 & 11,7 \\
\hline Otro & 7 & 1,3 \\
\hline Total & 549 & 100 \\
\hline
\end{tabular}

Fuente: historias clínicas institucionales

El mayor porcentaje de personas que consultaron no tienen afiliación a la seguridad social (vinculados, $n=152$; 27,7\%; tabla 2), con una mínima diferencia de quienes pertenecen al régimen contributivo $(n=150 ; 27,3 \%)$. 
Tabla 2. Afiliación a la seguridad social de los consultantes por trauma dentoalveolar. Universidad de Antioquia, Medellín, 2007-2012

\begin{tabular}{|l|r|c|}
\hline \multicolumn{1}{|c|}{ Régimen de seguridad social } & N. $^{\circ}$ & $\%$ \\
\hline No tiene & 55 & 10 \\
\hline Vinculado & 152 & 27,7 \\
\hline Subsidiado & 55 & 10 \\
\hline Contributivo & 150 & 27,3 \\
\hline Otro & 37 & 6,7 \\
\hline Sin dato & 100 & 18,2 \\
\hline Total & 549 & 100 \\
\hline
\end{tabular}

Fuente: historias clínicas institucionales

Las causas que dieron origen al trauma, en su mayoría, no fueron reportadas por los pacientes o por el clínico en la historia, en el momento de la atención en urgencias ( $\mathrm{n}=259 ; 47,2$; tabla 3 ); sin embargo, algunas causas reportadas fueron caídas $(\mathrm{n}=83 ; 15,1 \%)$ $\mathrm{y}$ actividades deportivas $(\mathrm{n}=37 ; 6,7 \%)$.

Otras causas reportadas como factor etiológico, aparte de los ítems principales, fueron al ingerir alimentos duros ( $\mathrm{n}=78 ; 14,2 \%)$, usando la bicicleta $(n=6 ; 1,1 \%)$, en accidentes caseros $(n=5 ; 0,9 \%)$, en episodios de epilepsia $(n=4 ; 0,7 \%)$, traumatismo con botellas $(n=3 ; 0,5 \%)$ y golpes con la cabeza $(n=3 ; 0,5 \%)$.
Tabla 3. Etiología del trauma dentoalveolar de los consultantes al servicio de urgencias. Universidad de Antioquia, Medellín, 2007-2012

\begin{tabular}{|l|r|r|}
\hline \multicolumn{1}{|c|}{ Causa del accidente } & N. ${ }^{\circ}$ & \multicolumn{1}{c|}{$\%$} \\
\hline Accidente de tránsito & 7 & 1,3 \\
\hline Accidente laboral & 3 & 0,5 \\
\hline Caída & 83 & 15,1 \\
\hline Hecho violento & 10 & 1,8 \\
\hline Violencia intrafamiliar & 2 & 0,4 \\
\hline Actividades deportivas & 37 & 6,7 \\
\hline Sin dato & 259 & 47,2 \\
\hline Otro & 148 & 27 \\
\hline Total & 549 & 100 \\
\hline
\end{tabular}

Fuente: historias clínicas institucionales

En los pacientes con trauma dentoalveolar, los principales diagnósticos encontrados (tabla 4) fueron: fractura no complicada de corona $(\mathrm{n}=310 ; 56,5 \%)$ y fractura complicada de corona $(\mathrm{n}=152 ; 27,7 \%)$. Así mismo, se encontró una prevalencia menor pero significativa de fractura de raíz $(\mathrm{n}=20 ; 3,6)$, fractura complicada de corona y raíz $(\mathrm{n}=18 ; 3,3 \%)$, subluxación $(\mathrm{n}=12 ; 2,2 \%)$ y avulsión $(\mathrm{n}=11 ; 2 \%)$. En los pacientes con más de un diente involucrado en el evento de trauma dentalveolar, se encontró que sigue siendo prevalente el diagnóstico de fractura no complicada de corona, fractura complicada de corona y avulsión.

Tabla 4. Diagnósticos de los dientes comprometidos por trauma dentoalveolar. Universidad de Antioquia, Medellín, 2007-2012

\begin{tabular}{|c|c|c|c|c|c|c|c|c|c|c|}
\hline \multirow[b]{2}{*}{ Diagnósticos } & \multicolumn{2}{|c|}{1 diente } & \multicolumn{2}{|c|}{2 dientes } & \multicolumn{2}{|c|}{3 dientes } & \multicolumn{2}{|c|}{4 dientes } & \multicolumn{2}{|c|}{5 dientes } \\
\hline & N. ${ }^{\circ}$ & $\%$ & N. ${ }^{\circ}$ & $\%$ & N. ${ }^{\circ}$ & $\%$ & N. ${ }^{\circ}$ & $\%$ & N. ${ }^{\circ}$ & $\%$ \\
\hline Fractura del esmalte & 3 & 0,5 & 1 & 0,2 & --- & --- & --- & --- & --- & --- \\
\hline Fractura no complicada de corona & 310 & 56,5 & 54 & 9,8 & 3 & 0,5 & 1 & 0,2 & --- & --- \\
\hline Fractura complicada de corona & 152 & 27,7 & 12 & 2,2 & 1 & 0,2 & 1 & 0,2 & --- & --- \\
\hline Fractura no complicada de corona y raíz & 2 & 0,4 & 1 & 0,2 & 1 & 0,2 & --- & --- & --- & --- \\
\hline Fractura complicada de corona y raíz & 18 & 3,3 & 2 & 0,4 & 1 & 0,2 & --- & --- & --- & --- \\
\hline Fractura de raíz & 20 & 3,6 & 1 & 0,2 & --- & --- & --- & --- & --- & --- \\
\hline Concusión & 5 & 0,9 & 5 & 0,9 & --- & --- & --- & --- & --- & --- \\
\hline Subluxación & 12 & 2,2 & 3 & 0,5 & 2 & 0,4 & 1 & 0,2 & 1 & 0,2 \\
\hline Luxación (sin especificar) & 2 & 0,4 & 1 & 0,2 & 1 & 0,2 & --- & --- & --- & --- \\
\hline Luxación lateral & 4 & 0,7 & --- & --- & --- & --- & --- & --- & --- & --- \\
\hline Luxación intrusiva & 9 & 1,6 & --- & --- & --- & --- & --- & --- & --- & --- \\
\hline Luxación extrusiva & 1 & 0,2 & 1 & 0,1 & --- & --- & --- & --- & --- & --- \\
\hline Avulsión & 11 & 2 & 7 & 1,3 & 4 & 0,7 & 1 & 0,2 & --- & --- \\
\hline Fractura del proceso mandibular & --- & --- & --- & --- & --- & --- & --- & --- & 1 & 0,2 \\
\hline No aplica & --- & --- & 461 & 84 & 536 & 97,6 & 545 & 99,2 & 547 & 99,6 \\
\hline Total & 549 & 100 & 549 & 100 & 549 & 100 & 549 & 100 & 549 & 100 \\
\hline
\end{tabular}

Fuente: historias clínicas institucionales 
Los dientes que más sufrieron trauma dentoalveolar fueron los anterosuperiores (tabla 5), afectando notablemente la estética, ya que fueron los dientes centrales superiores los que más estuvieron comprometidos (diente 11: $\mathrm{n}=180 ; 32,8 \%$ y diente $21: \mathrm{n}=141$; $25,7 \%)$, así como los dientes laterales, aunque en menor proporción que los centrales $(12: \mathrm{n}=43 ; 7,8 \%$ y 22 : $\mathrm{n}=27 ; 4,9 \%)$. Se evidenció que siguen siendo los anteriores superiores los que más sufren con estos accidentes. Es poco frecuente que se dé un evento de politraumatismo dentoalveolar ( $\mathrm{n}=458 ; 83,4 \%)$, es decir, en la ocurrencia del trauma dentoalveolar se afecta generalmente un solo diente.

Tabla 5. Dientes comprometidos, trauma dentoalveolar de consultantes. Universidad de Antioquia, Medellín, 2007-2012

\begin{tabular}{|c|c|c|c|c|c|c|c|c|c|c|}
\hline \multirow[b]{2}{*}{ Diente comprometido } & \multicolumn{2}{|c|}{1 diente } & \multicolumn{2}{|c|}{2 dientes } & \multicolumn{2}{|c|}{3 dientes } & \multicolumn{2}{|c|}{4 dientes } & \multicolumn{2}{|c|}{5 dientes } \\
\hline & N. ${ }^{\circ}$ & $\%$ & N. ${ }^{\circ}$ & $\%$ & N. ${ }^{\circ}$ & $\%$ & N. ${ }^{\circ}$ & $\%$ & N. ${ }^{\circ}$ & $\%$ \\
\hline 12 & 43 & 7,8 & 7 & 1,3 & $-{ }_{---}$ & --- & --- & --- & --- & $-\mathrm{c}^{---}$ \\
\hline 11 & 180 & 32,6 & $-\overline{c-}$ & --- & $-\overline{c-}$ & --- & --- & --- & --- & $-{ }_{---}$ \\
\hline 21 & 141 & 25,9 & 53 & 9,7 & 7 & 1,3 & 1 & 0,2 & --- & $-\mathrm{c}^{---}$ \\
\hline 22 & 27 & 4,9 & 8 & 1,5 & 3 & 0,5 & 2 & 0,4 & --- & $-{ }_{---}$ \\
\hline Otros dientes & 158 & 28,8 & 23 & 4,1 & 6 & 1,1 & 3 & 0,6 & 1 & 0,2 \\
\hline No aplica & 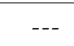 & $-\cdots$ & 458 & 83,4 & 533 & 97,1 & 543 & 98,8 & 548 & 99,8 \\
\hline Total & 549 & 100 & 549 & 100 & 549 & 100 & 549 & 100 & 549 & 100 \\
\hline
\end{tabular}

Fuente: historias clínicas institucionales

Al realizar la revisión de las historias clínicas de urgencias (tabla 6), se encontró que el ángulo mesial $(\mathrm{n}=74,13,5 \%)$ es la parte del diente más comprometida; sin embargo, también son afectadas las superficies incisal $(\mathrm{n}=46,8,4 \%)$ y el ángulo distal $(\mathrm{n}=34,6,2 \%)$, en el primer diente involucrado. Es de relevancia anotar que no se reportó en la historia clínica la superficie afectada en la mayoría de los casos; además, el ítem "No aplica" es una constante en aumento a partir del segundo diente, lo que indica variaciones en el diagnóstico de trauma dentoalveolar y poca frecuencia de politraumatismos.

Tabla 6. Superficie comprometida de dientes afectados por trauma dentoalveolar, Universidad de Antioquia, Medellín, 2007-2012

\begin{tabular}{|c|c|c|c|c|c|c|c|c|c|c|}
\hline \multirow[b]{2}{*}{ Parte del diente comprometido } & \multicolumn{2}{|c|}{1 diente } & \multicolumn{2}{|c|}{2 dientes } & \multicolumn{2}{|c|}{3 dientes } & \multicolumn{2}{|c|}{4 dientes } & \multicolumn{2}{|c|}{5 dientes } \\
\hline & N. ${ }^{\circ}$ & $\%$ & N. ${ }^{\circ}$ & $\%$ & N. ${ }^{\circ}$ & $\%$ & N. ${ }^{\circ}$ & $\%$ & N. ${ }^{\circ}$ & $\%$ \\
\hline Sin dato & 304 & 55,4 & 28 & 5,1 & 6 & 1,1 & 2 & 0,4 & --- & --- \\
\hline Ángulo mesial & 74 & 13,5 & 20 & 3,6 & 1 & 0,2 & --- & --- & --- & --- \\
\hline Incisal & 46 & 8,4 & 12 & 2,2 & --- & --- & --- & --- & --- & --- \\
\hline Ángulo distal & 34 & 6,2 & 7 & 1,3 & --- & --- & --- & --- & --- & --- \\
\hline Otros & 71 & 12,9 & 8 & 1,5 & 1 & 0,2 & 1 & 0,2 & 1 & 0,2 \\
\hline No aplica & 20 & 3,6 & 474 & 86,3 & 541 & 98,5 & 546 & 99,4 & 548 & 99,8 \\
\hline Total & 549 & 100 & 549 & 100 & 549 & 100 & 549 & 100 & 549 & 100 \\
\hline
\end{tabular}

Fuente: historias clínicas institucionales.

El principal tratamiento (tabla 7) para los pacientes que consultaron fue la obturación con resina de fotocurado $(\mathrm{n}=293,53,4 \%$ ), seguido de exodoncia $(\mathrm{n}=69,12,6 \%)$; se encontró también remisión a otra especialidad ( $\mathrm{n}=13 ; 2,3 \%)$, como endodoncia y prostodoncia. El ajuste oclusal es uno de los procedimientos que se realizaron para el manejo inicial del trauma $(\mathrm{n}=43 ; 7,8 \%)$. 
Tabla 7. Tratamiento realizado a los dientes afectados por trauma dentoalveolar, Universidad de Antioquia, Medellín, 2007-2012

\begin{tabular}{|c|c|c|c|c|c|c|c|c|c|c|}
\hline \multirow[b]{2}{*}{ Tratamiento realizado } & \multicolumn{2}{|c|}{1 diente } & \multicolumn{2}{|c|}{2 dientes } & \multicolumn{2}{|c|}{3 dientes } & \multicolumn{2}{|c|}{4 dientes } & \multicolumn{2}{|c|}{5 dientes } \\
\hline & N. ${ }^{\circ}$ & $\%$ & N. ${ }^{\circ}$ & $\%$ & N. ${ }^{\circ}$ & $\%$ & $\mathrm{~N} .^{\circ}$ & $\%$ & N. ${ }^{\circ}$ & $\%$ \\
\hline Obturación con resina & 293 & 53,4 & 56 & 10,2 & 3 & 0,54 & --- & --- & --- & --- \\
\hline Exodoncia & 69 & 12,6 & 11 & 2 & 3 & 0,54 & 1 & 0,2 & --- & --- \\
\hline Provisional & 67 & 12,2 & 1 & 0,18 & --- & --- & --- & --- & --- & --- \\
\hline Tratamiento pulpar & 37 & 6,7 & 8 & 1,4 & 1 & 0,2 & 2 & 0,4 & --- & --- \\
\hline Obturación temporal & 27 & 5 & --- & --- & --- & --- & --- & --- & --- & --- \\
\hline Remisión a otra especialidad & 13 & 2,3 & 1 & 0,18 & --- & --- & --- & --- & --- & --- \\
\hline Manejo farmacológico & --- & --- & 5 & 0,9 & 2 & 0,4 & 1 & 0,2 & --- & --- \\
\hline Ferulización & --- & --- & --- & --- & 3 & 0,54 & --- & --- & --- & --- \\
\hline Ninguno & --- & --- & --- & --- & 3 & 0,54 & --- & --- & --- & --- \\
\hline Sin dato & --- & --- & --- & --- & --- & --- & 1 & 0,2 & --- & --- \\
\hline Otros & 43 & 7,8 & 9 & 1,6 & --- & --- & --- & --- & --- & --- \\
\hline No aplica & --- & --- & 458 & 83,4 & 534 & 97,3 & 544 & 99 & 549 & 100 \\
\hline Total & 549 & 100 & 549 & 100 & 549 & 100 & 549 & 100 & 549 & 100 \\
\hline
\end{tabular}

Fuente: historias clínicas institucionales

En el análisis de los dientes involucrados en el trauma dentoalveolar, se tuvo en cuenta si estos dientes presentaban o no tratamiento endodóntico previo. Se encontró que, en su gran mayoría, no presentaban tratamiento previo $(n=333 ; 60,7 \%)$ en todos los dien- tes que sufrieron trauma dentoalveolar. Se sigue evidenciando la falta de registro del estado pulpar de los dientes involucrados en el evento de trauma en la historia clínica (tabla 8).

Tabla 8. Tratamiento endodóntico previo. Universidad de Antioquia, Medellín, 2007-2012

\begin{tabular}{|c|c|c|c|c|c|c|c|c|c|c|}
\hline & \multicolumn{2}{|c|}{1 diente } & \multicolumn{2}{|c|}{2 dientes } & \multicolumn{2}{|c|}{3 dientes } & \multicolumn{2}{|c|}{4 dientes } & \multicolumn{2}{|c|}{5 dientes } \\
\hline Tratamiento endodóntico previo & N. ${ }^{\circ}$ & $\%$ & N. ${ }^{\circ}$ & $\%$ & $\mathrm{~N} .^{\circ}$ & $\%$ & N. ${ }^{\circ}$ & $\%$ & N. ${ }^{\circ}$ & $\%$ \\
\hline Con tratamiento previo & 124 & 22,6 & 4 & 0,7 & --- & --- & --- & --- & --- & --- \\
\hline Sin tratamiento previo & 333 & 60,7 & 72 & 13,1 & 13 & 2,4 & 5 & 0,9 & 1 & 0,2 \\
\hline Sin dato & 92 & 16,8 & 14 & 2,6 & 3 & 0,5 & 1 & 0,2 & --- & --- \\
\hline No aplica & --- & --- & 459 & 83,6 & 533 & 97,1 & 543 & 98,9 & 548 & 99,8 \\
\hline Total & 549 & 100 & 549 & 100 & 549 & 100 & 549 & 100 & 549 & 100 \\
\hline
\end{tabular}

Fuente: historias clínicas institucionales

\section{Análisis bivariado}

Se exploró la existencia de asociación entre las siguientes variables: edad y causa del accidente, y se encontró asociación estadísticamente significativa $(p<0,05)$. Esto quiere decir que hay una relación entre la edad según la causa. Como se refiere en la revisión de tema, las causas del accidente del grupo etario menor de 20 años son distintas de las de personas de mayor edad.

$\mathrm{Al}$ analizar la edad con la ocupación de las personas que acudieron al servicio de urgencias, hay una relación significativa entre estas $(p<0,05)$, lo que muestra que, en determinadas edades, con cierto tipo de ocupaciones, puede existir relación directa con la ocurrencia del traumatismo dentoalveolar.
Cuando se analizaron las causas del accidente y el primer diente comprometido, se observó que no hay relación entre estas variables $(p>0,05)$, es decir, que la causa del accidente es independiente del diente que se puede afectar; esto mismo sucede entre la variable sexo y la causa del accidente $(p>0,05)$,

Finalmente, al relacionar la causa del accidente con los diagnósticos, vemos que entre el diagnóstico del diente afectado y la causa del accidente $(p<0,05)$ hay relación estadísticamente significativa, lo que puede indicar que la etiología del trauma podría determinar el diagnóstico del diente involucrado; sin embargo, cuando se relaciona con politraumatismos, no se encuentra asociación entre los demás diagnósticos con la causa del accidente. 


\section{Discusión de resultados}

El trauma dentoalveolar constituye uno de los más frecuentes motivos de consulta en los servicios de salud bucal, según lo demuestran los estudios revisados. El porcentaje de ocurrencia de este fenómeno es semejante al de otros países como Brasil o, incluso, al de algunos otros estudios reportados en Colombia. Si se compara la frecuencia de este incidente respecto al sexo de los pacientes atendidos, hay predominio del sexo femenino; situación diferente a las investigaciones realizadas en Recife (Brasil) [14], en la Clínica Universitaria de Alemania [21] y en el Sur de India [22], que indica mayor trauma dentoalveolar en hombres. Otro hallazgo diferente a nuestro estudio es la edad en la cual ocurre el incidente; mientras en diversos estudios se presenta con mayor frecuencia en edades tempranas $[3,6,9,14,21$ 23], nuestros resultados informan edades superiores a los 40 años. Este grupo de edad tiene como antecedentes una odontología más agresiva (extensión por prevención), además de la pérdida de minerales del esmalte.

No obstante, también se encuentran similitudes en varios de los estudios consultados [2, 8, 21-24] con respecto al motivo del trauma dentoalveolar, lo cual indica que la principal causa son los impactos directos por caídas, seguida de los accidentes automovilísticos, además del uso inadecuado de los dientes frente a objetos duros a la hora de la alimentación o simplemente asociados a hábitos [5].

Los dientes centrales superiores son, por excelencia, el grupo más comprometido en este tipo de episodios. Estos resultados están de acuerdo con los obtenidos en el estudio Pakistaní [25], en la Clínica Universitaria de Alemania [21] y en Suiza [26], que refieren estos mismos dientes como los de mayor compromiso. La superficie que más compromiso presentó fue la mesial, resultado acorde con la revisión de literatura en la Revista del Consejo de Odontólogos y Estomatólogos (RCOE) [6]. Como diagnóstico predominante, se obtuvo la fractura no complicada de corona, lo que concuerda con resultados obtenidos en la Clínica Universitaria de Alemania [21], en el estado de Santa Catarina, en Brasil [27] y en el Sur de India [22]. En cuanto al tratamiento, las restauraciones en resina ocupan el primer lugar, resultado que concuerda con los obtenidos en el estado de Santa Catarina, en Brasil [27], y en el sur del mismo país [28].

Los estudios analizados indican como factores predisponentes para la ocurrencia del trauma dentoalveolar un overjet mayor a $3 \mathrm{~mm}$ y la incompetencia labial [21, 22, 26, 28-30]. En este estudio no se encontró información correspondiente a estos aspectos en las historias clínicas. La literatura también indica que, luego de una lesión significativa que compromete otras estructuras faciales, los pacientes prefieren consultar primero en los servicios de medicina general [25], antes que solicitar atención odontológica; estos son resultados semejantes a los obtenidos en este estudio. Como consecuencia, los dientes pueden perder la vitalidad.

La aplicación de la vacuna antitetánica en pacientes comprometidos en el trauma dentoalveolar es un hallazgo de uno de los estudios consultados [25]. Nuestra investigación no revela datos acerca de este procedimiento.

\section{Conclusiones}

El trauma dentoalveolar es un evento común en la consulta odontológica que representa un desafío para el profesional a la hora de la atención clínica, por comprometer función y estética; sin embargo, el conocimiento que se tiene sobre el diagnóstico y manejo no cumple con los criterios propuestos por Andreasen [5].

Según esta investigación, el trauma dentoalveolar se presenta con mayor frecuencia en las mujeres mayores de 40 años, principalmente dientes anterosuperiores con fractura no complicada de corona.

La actual investigación corrobora como principales causas del trauma dentoalveolar las caídas, los impactos directos y el uso inadecuado de los dientes. Existen falencias desde la anamnesis y la descripción de las lesiones, hasta el diagnóstico de lesiones dentales y tejidos de soporte, lo que lleva a sugerir que se hace necesaria mayor rigurosidad en el diligenciamiento de la historia clínica.

Se sugiere desarrollar estudios sobre la incidencia de trauma dentoalveolar en dientes tratados endodónticamente, ya que se encontró una relación entre los dos eventos.

\section{Conflictos de interés}

Los autores somos integrantes de la comunidad académica de la institución donde se realizó el estudio.

\section{Referencias}

[1] León JC, Contreras E, Pinedo L, Galeano CF. Prevalencia del trauma dentoalveolar en pacientes atendidos en el servicio de urgencias de la Clínica Carlos Ardila Lulle de Floridablanca-Colombia, entre 1998 y 2002. Ustasalud Odontología. 2004;3:32-40. 
[2] Simões FG, Leonardi DP, Baratto Filho F, Ferreira EL, Fariniuk LF, Sayão SM. Fatores etiológicos relacionados ao traumatismo alvéolo-dentário de pacientes atendidos no pronto socorro odontológico do Hospital Universitário Cajuru. RSBO. 2004;1(1):50-5.

[3] García N, Legañoa J, Alonso C, Montalvo N. Comportamiento de los traumatismos dentoalveolares en niños y adolescentes. AMC. 2010;14(1):1-12.

[4] Berman LH, Blanco L, Cohen S. Manual clínico de traumatología dental. España: Elsevier; 2008.

[5] Andreasen JO, Andreasen FM, Andersson L. Texto y atlas a color de las lesiones traumáticas de las estructuras dentales. $4^{\mathrm{a}}$ ed. Colombia: AMOLCA. 2010.

[6] García-Ballesta C, Pérez L, Castejón I. Prevalencia y etiología de los traumatismos dentales. Una revisión. Madrid: RCOE. 2003;8(2):131-41.

[7] Bendo CB, Scarpelli AC, Vale MP, Araújo Zarzar PM. Correlation between socioeconomic indicators and traumatic dental injuries: a qualitative critical literature review. Dent Traumatol, 2009;25(4):420-5.

[8] Glendor U. Aetiology and risk factors related to traumatic dental injuries - a review of the literature. Dent Traumatol. 2009;25(1):19-31.

[9] Duque FL, Segura AM, Sánchez CA. Fracturas faciales en pacientes menores de veinte años atendidos en la unidad de cirugía maxilofacial y estomatología del Hospital Universitario San Vicente de Paúl y la Facultad de Odontología de la Universidad de Antioquia entre los años 1998 y 2007. Rev Fac Odontol Univ Antioq. 2010;22(1):40-9.

[10] Boix H, Gómez G, Sáez S, Bellet L. Consecuencias de traumatismos en dentición temporal sobre el germen del diente permanente en desarrollo. Rev Oper Dent Endod. 2007;5:76.

[11] Varela M, Botella JM, García-Camba JM, García-Hoyos F. Amelogénesis imperfecta: revisión. Cient Dent. 2008;5(3):239-46.

[12] Aldred MJ, Savarirayan R, Crawford PJM. La amelogénesis imperfecta: la clasificación y el catálogo para el siglo 21. Oral Dis. 2003;9(1):19-23.

[13] Arango A, Arbeláez A, Cárdenas D. Injurias traumáticas dentoalveolares en pacientes atendidos en la clínica Ces de Sabaneta, Antioquia, Colombia, entre 1992 y 1995. Ces Odontol. 2000;13(1):29-33.

[14] Leite A, Medeiros PK, Moraes D, Granville-García AF. Maxillofacial injuries and dental trauma in patients aged 19-80 years, Recife, Brazil. Rev Esp Cir Oral Maxilofac. 2010;32(1):11-6.

[15] Castro JC, Poi W, Manfrin TM, Zinza LG. Analysis of the crown fractures and crown-root fractures due to dental trauma assisted by Integrated Clinic from 1992 to 2002. Dent Traumatol. 2005;21(3):121-6.
[16] Souza FJ, Soares AJ. Avaliação das injúrias dentárias observadas no Centro de Trauma Dental da Faculdade de Odontologia de Piracicaba-Unicamp. RFO. 2009;14(2):111-6.

[17] Acevedo JP, Cooper H, Tirreau V, Núñez F. Descripción del traumatismo dentoalveolar en pacientes adultos. Rev Dental Chile. 2006;97(1):8-13.

[18] Hecova H, Tzigkounakis V, Merglova V, Netolicky J. A retrospective study of 889 injured permanent teeth. Dent Traumatol. 2010; 26(6):466-75.

[19] Prieto JL. Clasificación de los traumatismos dentales en paleopatología. Revista Paleopatología. 2006;33(1):1-6.

[20] Andreasen JO, Bakland LK, Flores MT, Andreasen FM, Andersson L. Manual de lesiones traumáticas dentarias. $3^{\text {a }}$ ed. España: Labor; 2012.

[21] Bücher K, Neumann C, Hickel R, Kühnisch J. Traumatic dental injuries at a German university clinic 20042008. Dent Traumatol. 2013;29(2):127-133.

[22] Ankola AV, Hebbal M, Sharma R, Nayak SS. Traumatic dental injuries in primary school children of South India. A report from district-wide oral health survey. Dent Traumatol. 2013;29(2):134-8.

[23] Targino AG, Queiroz L. Estudo o traumatismo dentario uma revisao crítica da literatura [monografía]. Paraíba: Universidade Federal da Paraíba; 2010.

[24] Caicedo R, Quintero OH, Méndez NR, Serpa MF. Injurias traumáticas dentoalveolares. Guías de práctica clínica basadas en la evidencia. Colombia: Instituto de Seguros Sociales; 1998.

[25] Khan SR, Din MU, Rehman MA, Nayyer K. Etiology, pattern and associated features of traumatic tooth fractures. Pak Oral \& Dental J. 2012;32(3):522-5.

[26] Schatz JP, Hakeberg M, Ostini E, Killaridis S. Prevalence of traumatic injuries to permanent dentition and its association with overjet in a Swiss child population. Dent Traumatol. 2013;29(2):110-4.

[27] Traebert J, Telino J, Foster LA, Thomson WM, Bortoluzzi MC. Impact of traumatic dental injuries on the quality of life of schoolchildren. Dent Traumatol. 2012;28(6):423-8.

[28] Schuch HS, Goettems ML, Correa MB, Torriani DD, Demarco FF. Prevalence and treatment demand after traumatic dental injury in South Brazilian schoolchildren. Dent Traumatol. 2013;29(4):297-302.

[29] De Vasconcelos Cunha Bonini GA, Marcenes W, Oliveira LB, Sheiham A, Bönecker M. Trends in the prevalence of traumatic dental injuries in Brazilian preschool children. Dent Traumatol. 2009;25(6):594-8.

[30] Damé-Teixeira N, Alves LS, Susin C, Maltz M. Traumatic dental injury among 12-year-old South Brazilian schoolchildren: prevalence, severity, and risk indicators. Dent Traumat. 2013;29(1):52-8. 